Schmerz 2022 - 36:13-18

https://doi.org/10.1007/s00482-021-00582-1

Angenommen: 26. Juli 2021

Online publiziert: 10. September 2021

(c) Der/die Autor(en) 2021

\section{Kritische Auseinandersetzung mit neuen Daten zur Prävalenz von Opioidgebrauchsstörungen bei Patienten mit chronischen Schmerzen in Deutschland}

\author{
Johannes Just ${ }^{1} \cdot$ F. Petzke ${ }^{2} \cdot$ N. Scherbaum ${ }^{3} \cdot$ L. Radbruch ${ }^{4} \cdot$ K. Weckbecker ${ }^{1}$. \\ W. Häuser, ${ }^{5,6}$ \\ ' Department für Humanmedizin, Institut für Allgemeinmedizin und ambulante Gesundheitsversorgung \\ (IAMAG), Universität Witten/Herdecke, Witten, Deutschland; ${ }^{2}$ Schmerzmedizin, Klinik für \\ Anästhesiologie, Universitätsmedizin Göttingen, Göttingen, Deutschland; ${ }^{3}$ LVR-Klinikum Essen, Klinik für \\ Psychiatrie und Psychotherapie, Kliniken der Universität Duisburg-Essen, Essen, Deutschland; ${ }^{4}$ Helios \\ Klinikum Bonn/Rhein-Sieg, Zentrum für Palliativmedizin, Akademisches Lehrkrankenhaus, Universität \\ Bonn, Bonn, Deutschland; ${ }^{5}$ Innere Medizin, Klinikum Saarbrücken, Saarbrücken, Deutschland; ${ }^{6}$ Klinik \\ für Psychosomatische Medizin und Psychotherapie der Technischen Universität München, München, \\ Deutschland
}

Zusammenfassung

Hintergrund: Es gibt keine Opioidkrise in Deutschland. Neue Studien mit Nichttumorpatienten mit chronischen Schmerzen (CNTS) in Deutschland zeigen jedoch eine unerwartet hohe Prävalenz von Opioidgebrauchsstörungen nach Diagnostic and Statistical Manual for Psychiatric Diseases 5 (DSM-5).

Ziel der Arbeit: Kritische Diskussion neuer Studienergebnisse zur Prävalenz von Opioidgebrauchsstörungen bei Schmerzpatienten in Deutschland.

Material und Methoden: Selektive Literaturrecherche und multiprofessionelle Einordnung der Ergebnisse durch Expertenrunde (Schmerztherapie, Neurologie, Psychiatrie, Palliativmedizin, Allgemeinmedizin und Suchttherapie).

Ergebnisse: Die Kriterien für die Diagnose „Opioidgebrauchsstörung" des DSM-5 sind auf Patienten mit CNTS nur eingeschränkt anwendbar, können aber für problematisches Verhalten sensibilisieren. Hierbei ist die Diagnose Opioidgebrauchsstörung nicht mit der Diagnose einer Substanzabhängigkeit nach ICD-10 gleichzusetzen, da die Diagnose nach DSM-5 ein deutlich breiteres Spektrum abdeckt (mild, moderat, schwer). Risikofaktoren für eine Opioidgebrauchsstörung sind jüngeres Alter, depressive Störungen, somatoforme Störungen und hohe Opioidtagesdosen. Die interdisziplinäre Leitlinie zur Langzeitanwendung von Opioiden bei chronischen nichttumorbedingten Schmerzen (LONTS) enthält Empfehlungen, welche das Risiko für eine Opioidgebrauchsstörung reduzieren sollen.

Diskussion: Eine Anpassung der DSM-5-Diagnosekriterien der Opioidgebrauchsstörung an die besondere Situation von Patienten mit CNTS und eine Validierung dieser Kriterien könnte helfen, in der Zukunft genauere Daten zu Opioidgebrauchsstörungen von Patienten mit chronischen Schmerzen in Deutschland zu erheben. Verordner sollten für diese Problematik sensibilisiert werden, ohne die Patienten zu pathologisieren oder gar zu stigmatisieren. Weitere Forschung zur Einordnung dieses bisher unterschätzten Phänomens ist notwendig.

\section{Schlüsselwörter}

Chronische nichttumorbedingte Schmerzen - Opioidmissbrauch · Opioidabhängigkeit · Rezeptierte Opioide · Leitlinie 
Tab. 1 Diagnosekriterien der Opioidgebrauchsstörung, Definition und Positivrate in den beiden zitierten Studien (Just et al. 2019 und Just et al. 2020)

\begin{tabular}{|l|l|l|}
\hline $\begin{array}{l}\text { Kriterien der Opioidgebrauchsstörung: } \\
\text { (Definition: problematisches Opioidkonsummuster, dass erhebliche } \\
\text { Beeinträchtigungen oder Stress verursacht) }\end{array}$ & $\begin{array}{l}\text { Rate positiver } \\
\text { Antworten (\%) }\end{array}$ \\
\cline { 2 - 3 } & $\begin{array}{l}\text { Studie 1 } \\
{[16]}\end{array}$ & $\begin{array}{l}\text { Studie 2 } \\
{[15]}\end{array}$ \\
\hline $\begin{array}{l}\text { 1. Die Substanz wird häufig in größeren Mengen oder über einen längeren } \\
\text { Zeitraum eingenommen als ursprünglich beabsichtigt }\end{array}$ & 13,5 & 9,4 \\
\hline $\begin{array}{l}\text { 2. Es besteht ein anhaltendes Verlangen oder ein nicht erfolgreicher Ver- } \\
\text { such, den Substanzkonsum zu kontrollieren oder zu reduzieren }\end{array}$ & 19,8 & 15,0 \\
\hline $\begin{array}{l}\text { 3. Ein großer Teil der Zeitspanne wird damit verbracht, die Substanz zu } \\
\text { besorgen, zu konsumieren oder sich von den Auswirkungen des Konsums } \\
\text { zu erholen }\end{array}$ & 7,6 & 5,2 \\
\hline $\begin{array}{l}\text { 4. Es besteht ein Craving (starkes Verlangen) oder ein Druck, die Substanz } \\
\text { zu konsumieren }\end{array}$ & 14,8 & 5,7 \\
\hline $\begin{array}{l}\text { 5. Wiederholter Substanzkonsum führt zum Versagen bei der Erfüllung } \\
\text { wichtiger Aufgaben (Rollenverpflichtungen im Beruf, in der Schule oder zu } \\
\text { Hause) }\end{array}$ & 11,0 & 20,8 \\
\hline $\begin{array}{l}\text { 6. Es besteht ein anhaltender Substanzkonsum ungeachtet der bestehen- } \\
\text { den oder wiederholt auftretenden sozialen oder interpersonellen Probleme, } \\
\text { die durch die Effekte des Substanzkonsums verursacht oder verstärkt wer- } \\
\text { den }\end{array}$ & 4,2 & 6,3 \\
\hline $\begin{array}{l}\text { 7. Relevante soziale, berufliche oder freizeitbezogene Aktivitäten werden } \\
\text { wegen des Substanzkonsums aufgegeben oder reduziert }\end{array}$ & 14,4 & 11,5 \\
\hline $\begin{array}{l}\text { 8. Die Substanz wird wiederholt in Situationen konsumiert, in denen dies } \\
\text { körperlich gefährlich ist }\end{array}$ & 5,9 & 14,9 \\
\hline $\begin{array}{l}\text { 9. Der Substanzkonsum wird ungeachtet des Wissens um bereits bestehen- } \\
\text { de oder wiederauftretende physische oder psychische Probleme fortge- } \\
\text { setzt, die sehr wahrscheinlich durch den Substanzkonsum verursacht oder } \\
\text { verstärkt werden }\end{array}$ & 8,9 & 3,6 \\
\hline $\begin{array}{l}\text { 2-3 Kriterien: mild, 4-5 Kriterien: moderat, } \geq 6 \text { Kriterien: schwere Störung } \\
\text { Kriterien 10 (Toleranzentwicklung) und 11 (Entzugssymptome) wurden gemäß Diagnosekriterien } \\
\text { nicht gezählt, da die untersuchten Patienten unter ärztlicher Therapiekontrolle waren }\end{array}$ & \\
\hline
\end{tabular}

\section{Der Stellenwert von Opioiden bei chronischen Schmerzen}

Opioidhaltige Analgetika gehören zur WHO-Liste der „essenziellen Medikamente". Opioidhaltige Schmerzmittel haben einen hohen Stellenwert in der Tumorschmerztherapie und Palliativmedizin [26]. Der Stellenwert von Opioiden bei chronischen Nichttumorschmerzen (CNTS) ist jedoch umstritten [2]. Opioidhaltige Schmerzmittel sind bezüglich der Schmerzreduktion nichtopioidhaltigen Schmerzmitteln und nichtmedikamentösen Therapieoptionen nicht überlegen [3]. Dennoch entfallen circa drei Viertel aller Opioidverschreibungen in Deutschland auf Patienten mit CNTS [19, 22].

\section{Gibt es eine Opioidepidemie in Deutschland?}

In der aktualisierten Leitlinie zur Langzeitanwendung von Opioiden bei chronischen

\section{Aktuelle Studien zur Prävalenz von Gebrauchsstörungen von rezeptierten Opioiden durch Patienten mit chronischen Schmerzen in Deutschland}

Im Juli 2020 erschien eine Analyse zu Gebrauchsstörungen von rezeptierten Opioiden durch Patienten mit chronischen Schmerzen in Deutschland, die auf den Daten des Suchtsurvey 2015 basiert [15]. Unter dem Titel "Rate of opioid use disorder in adults who received prescription opioid pain therapy-A secondary data analysis" beschreiben Just und Kollegen die Prävalenz von Opioidgebrauchsstörungen nach den Kriterien des DSM-5 in einer repräsentativen deutschen Kohorte (Kriterien für eine Opioidgebrauchsstörung siehe - Tab. 1). Von 9204 Studienteilnehmern hatten $n=275(3,5 \%) \mathrm{im}$ Jahr 2015 Opioide verschrieben bekommen. Davon erfüllten $n=54$ (21,2\%) die im Survey erfragten Diagnosekriterien für eine Opioidgebrauchsstörung nach DSM-5. Im Detail erfüllten 14,7\% die Kriterien für eine leichte Opioidgebrauchsstörung, weitere $3,5 \%$ für eine mittelgradige und 2,9\% für eine schwere Opioidgebrauchsstörung. Das Vorliegen einer Opioidgebrauchsstörung bei Opioidverschreibung war häufiger assoziiert mit psychiatrischen Erkrankungen, depressiven Störungen und dem Vorliegen nicht erklärbarer körperlicher Symptome und weniger häufig assoziiert mit dem Gebrauch nichtopioidhaltiger Analgetika. In einer kleineren, nicht repräsentativen Erhebung der DSM5-Kriterien bei Schmerzpatienten, die sich in schmerztherapeutischer Betreuung befanden (vier Praxen) und verschreibungspflichtige Opioide bereits länger als 6 Monate einnahmen, fiel die Rate an Opioidgebrauchsstörung mit $26,5 \%$ (leichtgradig: 17,2\% | mittelgradig: 4,4\% | hochgradig: 4,9\%) sogar noch etwas höher aus [16].

In einer weiteren Studie in Hausarztpraxen konnte bei 31,5\% der untersuchten $\mathrm{Pa}$ tienten, die verschreibungspflichtige Opioide bereits länger als 6 Monate einnahmen, ein "stark erhöhtes Risiko für Opioidmissbrauch" festgestellt werden [13]. Das hier verwendete Screeninginstrument, der COMM Score $^{\circledR}$ (Inflexxion Inc., Waltham, MA, USA), ist für die Nutzung in einem 
primärärztlichen Setting validiert und ist geeignet, Patienten mit einem „stark erhöhten Risiko für Opioidmissbrauch nach DSM-IV“ zu identifizieren. Die Testgüte in Bezug auf die DSM-IV-Diagnose „Opioidmissbrauch“ liegt dabei bei einer Sensitivität von $77 \%$ sowie einer Spezifität von $77 \%$ [20].

Eine Schwäche aller drei zitierten Studien ist die fehlende Erhebung der morphinäquivalenten Tagesdosis der genutzten Opioide sowie für die Studien [15] und [16] das Fehlen einer ärztlichen Kontrolle der erhobenen Diagnosekriterien.

\section{Widersprüche zwischen Prävalenz und Mortalität}

\section{Widersprüche zwischen Prävalenz und Mortalität}

Die hohe Rate von Opioidgebrauchsstörungen bei Patienten mit CNTS in Deutschland in beiden Studien steht scheinbar im Widerspruch zu der niedrigen Drogensterblichkeit und der niedrigen Rate von opioidabhängigen Personen in Deutschland. Während die jährliche Drogensterblichkeit in den USA 20/100.000 Einwohner beträgt, liegt sie in Deutschland lediglich bei 1,6/100.000 Einwohner. Auch die Prävalenz der Opiatabhängigkeit ist in Deutschland mit 0,2\% deutlich niedriger als in den USA mit $4 \%[1,18]$. Ebenso gibt es in Deutschland keine Berichte über Personen des öffentlichen Lebens, die von medikamentenassoziierten Todesfällen betroffen sind, ganz anders als in den USA, wo seit Jahren vermehrt berühmte Schauspieler, Musiker und Sportler an Überdosen verschreibungspflichtiger Opioide versterben [14]. Aber sowohl solche Fallbeschreibungen als auch die Todesursachenstatistik sind wegen Unterschieden in der Erhebungsmethodik nur begrenzt aussagekräftig und vergleichbar.

Gründe für die hohe Prävalenz von Opioidgebrauchsstörungen in den vorgestellten Studien

Aus unserer Sicht lassen sich die Differenzen zwischen Prävalenz und Mortalität aus unterschiedlichen Perspektiven erklären:

1. Definition und diagnostischer Rahmen im DSM-5: „Opioidgebrauchs- störung" ist definiert als „ein problematisches Opioidkonsummuster, dass erhebliche Beeinträchtigungen oder Stress verursacht". Das Ziel dieser neuen Definition war es, stigmatisierte Begriffe wie Sucht und Missbrauch zu vermeiden, aber auch das Spektrum opioidbezogener Störungen differenzierter abzubilden. Daher korrespondieren nur die Diagnosekategorien "mittelgradige" und "schwere" Opioidgebrauchsstörung mit den Missbrauchs- und Suchtkriterien in DSM-IV und ICD-10 [6]. Somit umfasst die "Opioidgebrauchsstörung" eine größere Patientengruppe, von der $6,4 \%$ Patienten mit mittel- bis schwergradiger Opioidgebrauchsstörung dem bisherigen Verständnis von Missbrauch/schädlichem Gebrauch und Abhängigkeit, wie es z. B. in der ICD-10 formuliert ist, entsprechen, während die 14,7\% mit einer leichten Opioidgebrauchsstörung Patienten sind, die aufgrund eines erhöhten Risikos ggf. lediglich eines intensivierteren Followups bedürfen.

2. Eine möglicherweise unzureichende Trennschärfe zwischen einer Opioidgebrauchsstörung und unzureichend behandelten Schmerzen im DSM-5: Es ist denkbar, dass bei Schmerzpatienten einzelne Diagnosekriterien im Rahmen des DSM-5 falsch interpretiert werden. So könnten zum Beispiel Kriterien wie „Opioide werden oft in größerer Menge oder über einen längeren Zeitraum als vorgesehen eingenommen" oder "Verlangen oder ein starker Wunsch, Opioide zu nehmen" auch Ausdruck von persistierenden oder eskalierenden Schmerzen sein und nicht Ausdruck einer Opioidgebrauchsstörung [10]. Eine Übersicht qualitativer Studien legte zudem nahe, dass Patienten auch unter ausreichender Opioidtherapie eine intensive Ambivalenz zwischen erlebter Wirkung, Nebenwirkungen und dem Gefühl der Notwendigkeit der Einnahme, als einer Form von Abhängigkeit, erleben [21]. Die Diagnose Opioidgebrauchsstörung ist definiert als ein problematisches Muster des Opioidkonsums, das erhebliche Beeinträchtigungen oder Stress verursacht ein solches problematisches Konsum- muster kann u. U. auch bei schwer zu beeinflussenden Schmerzen bestehen. Elander und Kollegen zeigten bei Patienten mit chronischen Schmerzen bei Sichelzellanämie eine deutliche Reduktion der Häufigkeit der Diagnose Opioidmissbrauch und -abhängigkeit nach DSM-IV, wenn für den Umstand einer schmerzbedingt positiven Antwort korrigiert wurde [8]. Daher ist weitere Forschung nötig, um zu prüfen, ob einzelne Diagnosekriterien des DSM- 5 bei chronischen Schmerzpatienten anders oder sogar überhaupt nicht genutzt werden sollten.

3. Die besondere wirtschaftliche, gesellschaftliche und soziale Situation in den USA: Wahrscheinlich spielen bei der Entwicklung des nationalen Gesundheitsnotstands in den USA („Opioidkrise" oder "Opioidepidemie“) gesellschaftliche Faktoren eine große Rolle, die nicht auf andere Staaten generalisiert werden können. Daher ist es möglich, dass es auch in Deutschland bei Patienten mit Verschreibung von Opioidanalgetika Opioidgebrauchsstörungen gibt, ohne dass sich daraus ein nationaler Gesundheitsnotstand entwickeln müsste. Die Ökonomen Anne Case und Angus Deaton beschreiben in ihrem 2020 erschienenen Buch Deaths of Despair die Krise der amerikanischen Mittelschicht und deren verheerenden Einfluss auf die Lebenserwartung in den USA. Die USA sind das einzige industrialisierte Land der Welt, in dem die Lebenserwartung im 21. Jahrhundert gesunken ist. Hauptsächlich lässt sich dieser Effekt auf eine Gruppe zurückführen, die Case und Deaton als "Verzweiflungstote" bezeichnen, die Opfer von Selbstmord, Alkoholabhängigkeit und Opioidüberdosierungen. Dieser Effekt findet sich in den USA allerdings nur in einer Bevölkerungsschicht in dieser Deutlichkeit: der weißen, nichtakademischen Mittelschicht. Case und Deaton erklären diese "Verzweiflungstoten" durch Arbeitslosigkeit und Kaufkraftverlust in dieser Schicht, die besonders stark durch die Abwanderung und den Abbau von Arbeitsstellen in der produzierenden Industrie betroffen ist. Da solidarische Sicherungssysteme 
Tab. 2 Auffälliges Verhalten bei Schmerzpatienten

\begin{tabular}{|c|c|c|}
\hline $\begin{array}{l}\text { Nichtspezifische Hinweise } \\
\text { für Fehlgebrauch }\end{array}$ & $\begin{array}{l}\text { Nichtspezifische Hinweise für Missbrauch/ } \\
\text { Abhängigkeit }\end{array}$ & Spezifischere Hinweise auf Abhängigkeit \\
\hline $\begin{array}{l}\text { 口Einnahme trotz geringer } \\
\text { bis fehlender Wirksamkeit } \\
\text { 口Ein Fehlgebrauch, der } \\
\text { trotz Aufklärung, Abspra- } \\
\text { che und engerer ärztli- } \\
\text { cher Anbindung anhält, } \\
\text { kann als Hinweis auf einen } \\
\text { schädlichen Gebrauch/ } \\
\text { Abhängigkeit gewertet } \\
\text { werden } \\
\text { घWechselnde Schmerzlo- } \\
\text { kalisationen, multilokuläre } \\
\text { Ausbreitung (Generali- } \\
\text { sierung) der Schmerzen, } \\
\text { Transformation des Primär- } \\
\text { schmerzes unter laufender } \\
\text { Therapie } \\
\text { —Opioidinduzierte Hy- } \\
\text { peralgesie (Tendenz zur } \\
\text { Schmerzausbreitung, Er- } \\
\text { höhung der Schmerzemp- } \\
\text { findlichkeit und Opioidresis- } \\
\text { tenz) }\end{array}$ & 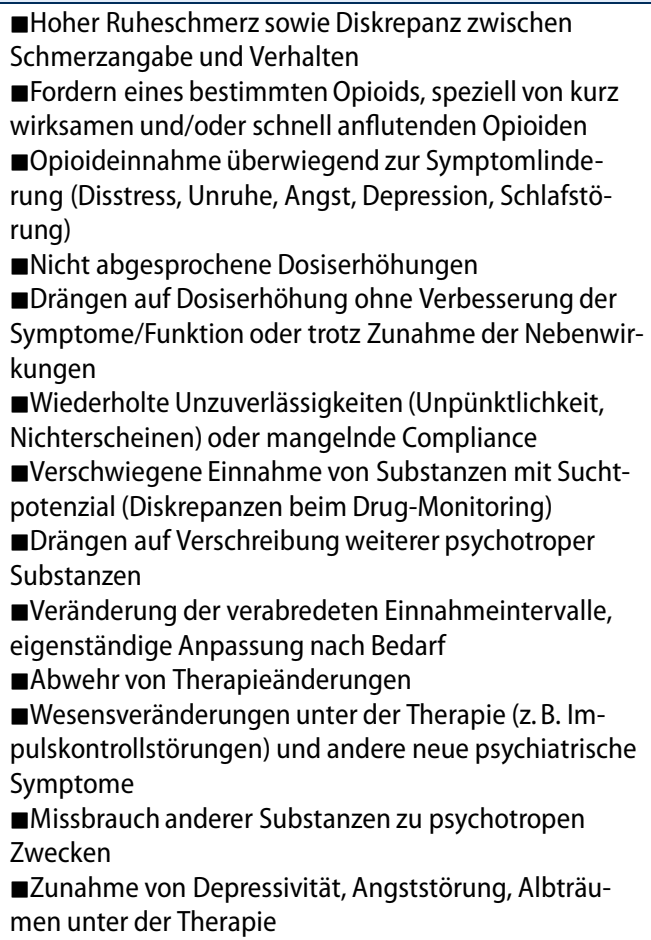 & 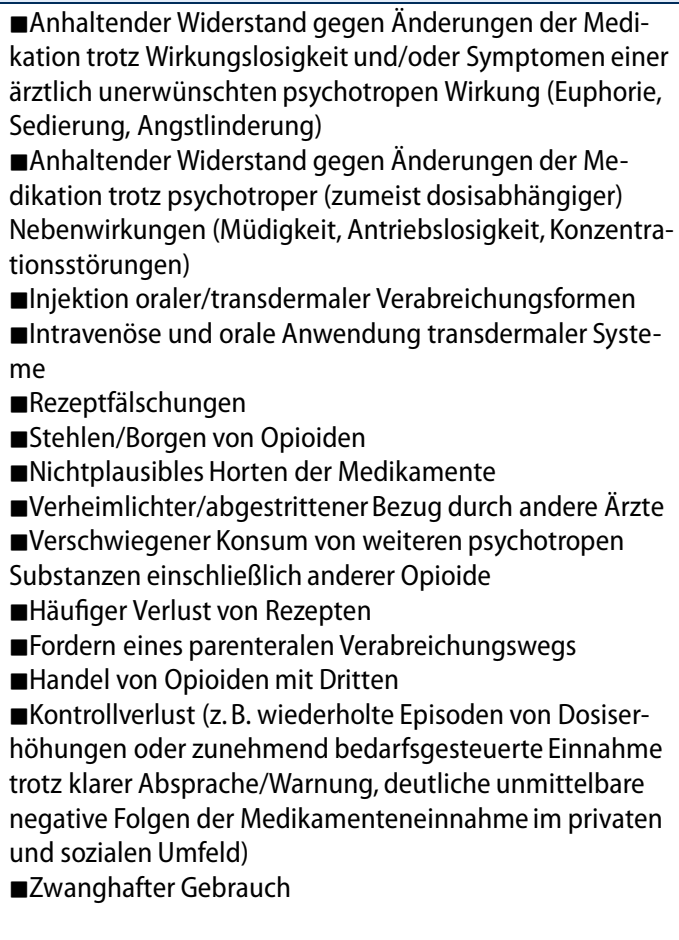 \\
\hline
\end{tabular}

in den USA nur minimal ausgeprägt sind und von Betroffenen teilweise sogar ideologisch abgelehnt werden, konnte dieser soziale und ökonomische Abstieg nicht abgefedert werden [4]. Als weiterer USA-spezifischer Faktor ist das in der Vergangenheit sehr aggressive Marketingverhalten einzelner Opioidhersteller zu nennen. Neben dem direkten Konsumentenmarketing (Werbung am Patienten, in Deutschland nicht erlaubt) trugen auch gezielte Fehlbehauptungen zur Entwicklung der Opioidkrise in den USA bei [9]. Zu den Fehlbehauptungen über ihr Produkt „Oxycontin“, zu denen sich die Firma Purdue Pharma bekannt hat, gehört unter anderem, dass es nicht euphorisierend wirke und keine Gewöhnungs- oder Entzugseffekte auftreten. Im Zusammenhang von hohen Vergleichszahlungen meldete die Firma 2019 Konkurs an [5, 27].

4. Die besondere wirtschaftliche, gesellschaftliche und soziale Situation in Deutschland: Das Vorhandensein und die hohe Qualität sozialer und solidarischer Sicherungssysteme in Deutschland ist wahrscheinlich ein wichtiger Einflussfaktor, der die Entwicklung von der Opioidgebrauchsstörung zur Opioidabhängigkeit und zum Tod durch Überdosis hemmt. Qualitativ hochwertige ambulante und stationäre Versorgungsangebote sind für Menschen in Deutschland meist niederschwellig erreichbar. Hierzu zählt die primärärztliche Grundversorgung, aber auch spezialisierte Angebote wie Schmerztherapie, suchtmedizinische Grundversorgung, Psychotherapie und spezialisierte psychiatrische Angebote [7].

\section{Prävention}

Es bleibt die kommunikative Herausforderung, wie eine angemessene Prävention und Erkennung von Opioidgebrauchsstörungen in Deutschland erfolgen kann, ohne dadurch unnötige Sorgen vor Opioidnebenwirkungen bei Ärzten und Patienten zu schüren.

Der Schlüssel zur erfolgreichen Prävention liegt zum einen in der richtigen Indikation, Dosierung und Therapiedauer und zum anderen im frühzeitigen Erkennen potenziell gefährdeter Patienten.
Dies beinhaltet laut LONTS-Leitlinie konkret: a) das Screening auf psychische und substanzbezogene Störungen und gegebenenfalls eine fachpsychotherapeutische/psychiatrische Vorstellung vor Beginn einer Therapie mit Opioiden, b) das Beachten von Kontraindikationen (z.B. psychische Störungen mit Leitsymptom Schmerz), c) das Festlegen von Therapiezielen, d) die Beendigung der Therapie, wenn die Therapieziele nicht erreicht sind, und e) regelmäßige Evaluation der Wirksamkeit und Nebenwirkungen inklusive Hinweise missbräuchlicher Verwendung [11].

\section{Erkennen einer Opioidgebrauchs- störung}

Die bisher für die deutsche Bevölkerung beschriebenen Risikoindikatoren für eine Opioidgebrauchsstörung bzw. Missbrauch/Abhängigkeit von rezeptierten Opioiden sind:

- Jüngeres Lebensalter: OR 0,96 $(95 \% \mathrm{Cl}$ $0,95 ; 0,97 ;[19])$ sowie OR $0,96(95 \% \mathrm{Cl}$ $0,94 ; 0,99 ;[16])$

- Somatoforme Schmerzstörung: OR $1,92(95 \% \mathrm{Cl} 1,18 ; 23,16)$ und OR 
1,89 (95\% Cl 1,56; 2,28; [12, 19]) bzw. unklare körperliche Beschwerden OR 2,68 (95\% CI 1,14; 6,31; [15])

- Depressive Störungen: OR 2,26 $(95 \% \mathrm{Cl}$ $1,37 ; 6,58)$, OR $2,52(95 \% \mathrm{Cl} 2,12 ; 3,00)$ und OR 2,69 (95\% Cl 1,13; 6,38; [12, 15 , 19])

- Hochdosis-LTOT (> 120 mg morphinäquivalente Tagesdosis) $1,81(95 \% \mathrm{Cl}$ $1,44 ; 2,27 ;[12])$

- Psychiatrische Diagnose OR 4,12 (95\% Cl 1,36; 12,43; [15])

Eine Vorgeschichte von einer substanzbezogenen Störung, z.B. Alkoholabhängigkeit, ist ein, im internationalen Setting, häufig nachgewiesener und als sehr relevant eingestufter Risikofaktor, der in Deutschland bisher nicht nachgewiesen wurde, aber mutmaßlich auch hier eine große Rolle spielt [23, 24]. Die LONTS-Leitlinie stellt zudem eine Aufzählung auffälliger Verhaltensmuster einer missbräuchlichen/abhängigen Verwendung zur Verfügung, welche in den diagnostischen Kriterien von DSM-IV, DSM-5, ICD-10 und ICD11 nicht enthalten sind, deren Anwendung klinisch aber sehr hilfreich sein kann (- Tab. 2, [11]).

\section{Klinische Evaluation potenziell betroffener Patienten}

Bisher gibt es kein ausreichend validiertes und geeignetes Screeninginstrument für das Vorliegen opioidassoziierter Probleme, das in der Breite der Patientenversorgung in Deutschland uneingeschränkt empfohlen werden könnte. Die besondere Stärke der DSM-5-Diagnosekriterien für eine Opioidgebrauchsstörung liegt aus unserer Sicht darin, dass zum einen ein dynamisches Spektrum abgebildet wird (leicht, mittel, schwer), und zum anderen stigmatisierende Begriffe wie Abhängigkeit und Sucht vermieden werden. Andererseits gibt es berechtigte Zweifel bezüglich der Anwendbarkeit einzelner Kriterien bei Patienten mit chronischen Schmerzen. Daher kann die klinische Nutzung aktuell nicht uneingeschränkt empfohlen werden, zumal in Deutschland die ICD-10/11 und nicht der DSM-5 den diagnostischen Codierungsstandard darstellt. Somit stellt die aufmerksame klinische Beobachtung des Patienten un- ter anderem unter Berücksichtigung der genannten Risikoindikatoren und auffälliger Verhaltensmuster den Goldstandard der Erkennung betroffener Patienten dar. Ein weiterer zentraler Teil der Evaluation sollte immer die Frage sein, ob die betroffenen Schmerzpatienten aktuell mehr unter der Wirkung und Nebenwirkung der eingenommenen Opioide leiden, als sie durch eine Schmerzreduktion profitieren. Dabei gilt es zu prüfen, welche weiteren Maßnahmen der multimodalen Schmerztherapie noch ausgeschöpft werden können. Bei gegebener Indikation sind auch Maßnahmen zur Verbesserung der problematischen Anwendung (Edukation, engmaschigere Betreuung, Vereinbarung gemeinsamer Regeln zur Verordnung) sinnvoll. Erst wenn wiederholt Probleme mit Entzugssymptomen oder problematischem Verhalten des Patienten auftreten, ist eine suchtmedizinische/suchtpsychiatrische Vorstellung/ Mitbehandlung dringend zu erwägen [11, 17].

\section{Fazit für die Praxis}

- Mittel- bis schwergradige Opioidgebrauchsstörungen nach DSM-5 zeigen eine gute Korrelation mit den bekannten Diagnosen „Abhängigkeit" sowie "Missbrauch" bzw. "schädlicher Gebrauch" in DSM-IV und ICD-10. Die Prävalenz in Deutschland liegt wahrscheinlich im höheren einstelligen Bereich.

- Patienten jüngeren Alters mit depressiven Störungen, mit somatoformen Störungen und hohen Opioidtagesdosen sind besonders gefährdet, Opioidgebrauchsstörungen bzw. Missbrauch/Abhängigkeit zu entwickeln.

- Da Zweifel daran bestehen, ob alle Diagnosekriterien von DSM-IV, DSM-5 und ICD-10/11 sinnvoll bei Patienten mit CNTS angewendet werden können, brauchen wir weitere Forschung zur Anwendbarkeit der genannten Diagnosekriterien.

- Das aufmerksame ärztliche Gespräch unter Beachtung bekannter Risikofaktoren und auffälligen Verhaltens ist aktuell der diagnostische Goldstandard zur Erkennung von Patienten mit opioidassoziierten Problemen.

- Die Leitlinien „LONTS Update“ und „Medikamentenbezogene Störung" bieten Hinweise zum weiteren Umgang mit betroffenen Patienten.

\section{Korrespondenzadresse}

\section{Dr. med. Johannes Just}

Department für Humanmedizin, Institut für Allgemeinmedizin und ambulante Gesundheitsversorgung (IAMAG), Universität Witten/Herdecke

Alfred-Herrhausen-Str. 50, 58448 Witten, Deutschland

johannes.just@uni-wh.de

Funding. Open Access funding enabled and organized by Projekt DEAL.

\section{Einhaltung ethischer Richtlinien}

Interessenkonflikt. J. Just, F. Petzke, L. Radbruch, K. Weckbecker und W. Häuser geben an, dass kein Interessenkonflikt besteht. N. Scherbaum hat für Tätigkeiten in Advisory Boards, Vortragstätigkeit, Erstellung von Manuskripten sowie Erstellung von Weiterbildungsmaterial Honorare von den Firmen AbbVie, Camurus, Hexal, Janssen-Cilag, Lundbeck, MSD, Medice, Mundipharma, Reckitt-Benckiser/Indivior und Sanofi-Aventis erhalten. Er hat in den letzten 3 Jahren an Medikamentenprüfungen teilgenommen, die von der pharmazeutischen Industrie finanziert wurden.

Für diesen Beitrag wurden von den Autoren keine Studien an Menschen oder Tieren durchgeführt. Für die aufgeführten Studien gelten die jeweils dort angegebenen ethischen Richtlinien.

Open Access. Dieser Artikel wird unter der Creative Commons Namensnennung 4.0 International Lizenz veröffentlicht, welche die Nutzung, Vervielfältigung, Bearbeitung, Verbreitung und Wiedergabe in jeglichem Medium und Format erlaubt, sofern Sie den/die ursprünglichen Autor(en) und die Quelle ordnungsgemäß nennen, einen Link zur Creative Commons Lizenz beifügen und angeben, ob Änderungen vorgenommen wurden.

Die in diesem Artikel enthaltenen Bilder und sonstiges Drittmaterial unterliegen ebenfalls der genannten Creative Commons Lizenz, sofern sich aus der Abbildungslegende nichts anderes ergibt. Sofern das betreffende Material nicht unter der genannten Creative Commons Lizenz steht und die betreffende Handlung nicht nach gesetzlichen Vorschriften erlaubt ist, ist für die oben aufgeführten Weiterverwendungen des $\mathrm{Ma}$ terials die Einwilligung des jeweiligen Rechteinhabers einzuholen.

Weitere Details zur Lizenz entnehmen Sie bitte der Lizenzinformation aufhttp://creativecommons.org/ licenses/by/4.0/deed.de.

\section{Literatur}

1. Ashburn MA, Fleisher LA (2018) Increasing evidence for the limited role of Opioids to treat chronic noncancer pain. JAMA 320:2427-2428

2. Bialas P, Maier C, Klose P, Häuser W (2020) Efficacy and harms of long-term opioid therapy in chronic non-cancer pain: systematic review and metaanalysis of open-label extension trials with a study duration $\geq 26$ weeks. Eur JPain 24:265-278 
3. Busse JW, Wang L, Kamaleldin M et al (2018) Opioids for chronic noncancer pain: a systematic review and meta-analysis. JAMA 320:2448-2460

4. Case A, Deaton A (2020) Deaths of despair and the future of capitalism https://doi.org/10.2307/j. ctvpr7rb2

5. Contributors to Wikimedia projects (2005) Purdue Pharma. https://en.wikipedia.org/wiki/Purdue_ Pharma.Zugegriffen:9.Jan. 2021

6. Degenhardt L, Bruno R, Lintzeris N et al (2015) Agreement between definitions of pharmaceutical opioid use disorders and dependence in people taking opioids for chronicnon-cancerpain (POINT): a cohort study. Lancet Psychiatry 2:314-322

7. Deutsche Gesellschaft für Psychiatrie und Psychotherapie, Psychosomatik und Nervenheilkunde (DGPPN), Deutsche Gesellschaft für Suchtforschung und Suchttherapie (DG-Sucht) (2020) S3Leitlinie Medikamentenbezogene Störungen - 1. Auflage. Version 08.2020

8. Elander J, Lusher J, Bevan D, Telfer P (2003) Pain management and symptoms of substance dependence among patients with sickle cell disease. Soc Sci Med 57:1683-1696

9. Fischer B, Keates A, Bühringer G et al (2014) Nonmedical use of prescription opioids and prescription opioid-related harms: why so markedly higher in North America compared to the rest of the world? Addiction 109:177-181

10. Gorfinkel L, Voon P, Wood E, Klimas J (2018) Diagnosing opioid addiction in people with chronic pain. BMJ. https://doi.org/10.1136/bmj. k3949

11. Häuser W, Koautoren für die Konsensusgruppe der 2.Aktualisierung derS3-Leitlinie LONTS, BockFetal (2020) Empfehlungen der zweiten Aktualisierung der Leitlinie LONTS. Der Schmerz 34:204-244. https://doi.org/10.1007/s00482-020-00472-y

12. Häuser W, Schubert T, Scherbaum N, Tölle T (2018) Langzeitopioidtherapie von nichttumorbedingten Schmerzen. Schmerz 32:419-426. https://doi. org/10.1007/s00482-018-0324-4

13. Just JM, Bingener $L$, Bleckwenn $M$ et al (2018) Risk of opioid misuse in chronic non-cancer pain in primary care patients-a cross sectional study. BMCFam Pract 19:92

14. Just JM, Bleckwenn M, Schnakenberg Ret al (2016) Drug-related celebrity deaths: a cross-sectional study. Subst Abuse Treat Prev Policy. https://doi. org/10.1186/s13011-016-0084-z

15. Just JM, Scherbaum N, Specka M et al (2020) Rate of opioid use disorder in adults who received prescription opioid pain therapy-A secondary data analysis. PLoSONE 15:e236268

16. Just JM, Schwerbrock F, Bleckwenn M et al (2019) Opioid use disorder in chronic non-cancer pain in Germany: a cross sectional study. BMJ Open 9:e26871

17. Just J, Mücke M, Bleckwenn M (2016) Dependence on prescription opioids: prevention, diagnosis and treatment. Dtsch Aerztebl. https://doi.org/10. 3238/arztebl.2016.0213

18. Kraus L, Seitz N-N, Schulte B et al (2019) Estimation of the number of people with opioid addiction in Germany. Dtsch Arztebl Int 116:137-143

19. MarschallU, L'hoestH, Radbruch L, HäuserW(2016) Long-term opioid therapy for chronic non-cancer pain in Germany. Eur J Pain 20:767-776. https:// doi.org/10.1002/ejp.802

20. Meltzer EC, Rybin D, Saitz R et al (2011) Identifying prescription opioid use disorder in primary care: diagnostic characteristics of the Current Opioid Misuse Measure (COMM). Pain 152:397-402

\section{Critical discussion of new data regarding prevalence of opioid use disorder in patients with chronic pain in Germany}

Background: There is no opioid crisis in Germany. However, new studies involving patients with chronic noncancer pain (CNCP) in Germany show an unexpectedly high prevalence of opioid use disorder according to DSM-5 (Diagnostic and Statistical Manual for Psychiatric Diseases).

Objectives: Critical discussion of new study results on the prevalence of opioid use disorder in CNCP patients in Germany.

Materials and methods: Selective literature search and multiprofessional classification of results by an expert panel (pain therapy, neurology, psychiatry, palliative medicine, general medicine and addiction therapy).

Results: The DSM-5 criteria for the diagnosis of "opioid use disorder" have limited applicability to patients with CNCP, but may raise awareness of problematic behavior. The diagnosis of opioid use disorder is not the same as the diagnosis of substance dependence according to ICD-10, as the DSM-5 diagnosis covers a much broader spectrum (mild, moderate, severe). Risk factors for opioid use disorder include younger age, depressive disorders, somatoform disorders, and high daily opioid doses. The interdisciplinary guideline on long-term opioid use for CNCP (LONTS) includes recommendations intended to reduce the risk for opioid use disorder.

Conclusion: An adaptation of the DSM-5 diagnostic criteria of opioid use disorder to the specific situation of CNCP patients and a validation of these criteria could help to collect more accurate data on opioid use disorders of patients with chronic pain in Germany in the future. Prescribers should be sensitized to this problem without pathologizing or even stigmatizing patients. Further research is needed to classify this previously underestimated phenomenon.

\section{Keywords}

Chronic noncancer pain · Opioid misuse · Opioid dependence · Prescribed opioids · Guideline

21. Nichols VP, Toye F, Eldabe S et al (2020) Experiences of people taking opioid medication for chronic non-malignant pain: a qualitative evidence synthesis using meta-ethnography. BMJ Open 10:e32988

22. Schubert I, Ihle P, Sabatowski R (2013) Increase in opiate prescription in Germany between 2000 and 2010: a study based on insurance data. Dtsch Arztebl Int 110:45-51

23. Turk DC, Swanson KS, Gatchel RJ (2008) Predicting opioid misuse by chronic pain patients. Clin J Pain 24:497-508. https://doi.org/10.1097/ajp. Ob013e31816b1070

24. Zegel M, Rogers AH, Vujanovic AA, Zvolensky MJ (2020) Alcohol use problems and opioid misuse and dependence among adults with chronic pain: the role of distress tolerance. Psychol Addict Behav. https://doi.org/10.1037/adb0000587

25. Zenz M (2020) How long does a long-term therapy last? Schmerz 34:438-442

26. WHO model list of essential medicines. https://www.who.int/publications/i/item/ WHOMVPEMPIAU2019.06. Zugegriffen: 23. Nov. 2020

27. U.S. v. PURDUE FREDERICK CO., INC (2007) https://www.casemine.com/judgement/us/ 59146d9aadd7b049343266a9. Zugegriffen: 24. Nov. 2020 